\title{
Campylobacter jejuni: A rare agent in a child with peritoneal dialysis-related peritonitis
}

\author{
Tugce Tural Kara M.D. ${ }^{a}$, Songul Yilmaz M.D. ${ }^{b}$, Halil Ozdemir M.D. ${ }^{a}$, Zeynep Birsin Ozcakar M.D. ${ }^{b}$, \\ Ahmet Derya Aysev M.D. ${ }^{c}$, Ergin Ciftci M.D. ${ }^{a}$ and Erdal Ince M.D. ${ }^{a}$
}

\begin{abstract}
Peritonitis is a serious problem in children receiving peritoneal dialysis. Campylobacter jejuni is an unusual cause of peritonitis. A 10-year-old boy who had end stage renal failure due to atypical hemolytic uremic syndrome was admitted to our hospital with abdominal pain and fever. Peritoneal dialysis fluid was cloudy and microscopic examination showed abundant leukocytes. Intraperitoneal cefepime treatment was started. Campylobacter jejuni was isolated from peritoneal dialysis fluid culture and oral clarithromycin was added to the treatment. At the end of therapy, peritoneal fluid culture was negative. To our knowledge, C. jejuni peritonitis was not reported in children previously.

Conclusion: Although C. jejuni peritonitis is rarely encountered in children, it should be considered as an etiologic factor for peritonitis.

Key words: Campylobacter jejuni; peritoneal dialysis, continuous ambulatory; peritonitis.
\end{abstract}

http:/ / dx.doi.org/10.5546/aap.2016.eng.e354

\section{INTRODUCTION}

Peritonitis is a major complication of peritoneal dialysis. Staphylococcus epidermidis, Staphylococcus aureus, Streptococcus spp., Enterococcus faecalis and Escherichia coli are the most common causative pathogens for peritonitis. ${ }^{1}$ Guidelines recommend that selection of empiric therapy must cover both gram-positive and gram-negative microorganisms and a fourth-generation cephalosporin is a suitable agent. In addition a first-generation cefalosporin may be used with ceftazidime or aminoglycoside, if cefepime is not available. After

a. Department of Pediatric Infectious Diseases

b. Department of Pediatric Nephrology,

c. Department of Microbiology Laboratory,

Ankara University Medical School, Ankara, Turkey.

E-mail Address:

Tugce Tural Kara: tugcetural@hotmail.com

Funding: None.

Conflict of interest: None.

Received: 1-19-2016

Accepted: 2-17-2016 causative organism is identified, treatment should be organized according to microorganism. ${ }^{2}$ Minimum length of therapy is two weeks, for severe infections the recommended treatment duration is three weeks. ${ }^{3}$

In literature Campylobacter peritonitis has been reported in adult patients. In this report we present the first pediatric case with $C$. jejuni peritonitis.

\section{CASE REPORT}

A 10-year-old boy with end stage renal failure secondary to atypical hemolytic uremic syndrome was treated with continuous ambulatory peritoneal dialysis (CAPD) for 6 years. Methicillin resistant Staphylococcus epidermidis (MRSE) peritonitis occured in $3^{\text {rd }}$ month of dialysis treatment. Intravenous vancomycin and intraperitoneal cefazolin were used for therapy. Staphylococcus epidermidis peritonitis developed 12 months later. Four years after the second peritonitis, he admitted to hospital with abdominal pain and fever. The patient did not declare a complaint of diarrhea or constipation He reported ultrafiltration failure and peritoneal dialysis fluid was cloudy in the last 1 week. Physical examination revealed; temperature: $38.1 \mathrm{C}^{\circ}$, heart rate: 118 beats / minute, blood pressure: $130 / 85 \mathrm{mmHg}$, body weight: 34.5 $\mathrm{kg}$ (previous weight: $25 \mathrm{~kg}$ ) and generalized edema. Ascites and tenderness were found in the abdomen. There were no signs of exitsite infection. Laboratory test results were as follows; haemoglobin: $9.4 \mathrm{~g} / \mathrm{dl}$, leukocyte count: $9500 / \mathrm{mm}^{3}$, platelet count: $183000 / \mathrm{mm}^{3}$, blood urea nitrogen: $91 \mathrm{mg} / \mathrm{dl}$, creatinine: $7.09 \mathrm{mg} /$ dl, C-reactive protein: $78.2 \mathrm{mg} / \mathrm{L}$, erythrocyte sedimantation rate: $62 \mathrm{~mm} / \mathrm{h}$. Microscopic examination of the peritoneal dialysis fluid showed abundant leukocytes with the diagnosis of peritonitis. Intraperitoneal cefepime treatment was started empirically. Temporary hemodialysis was performed due to fluid overload. On the $3^{\text {rd }}$ day of treatment clinical and laboratory findings improved, patient's temperature was normal and peritoneal dialysis fluid became clear. 
C. jejuni was isolated from the peritoneal fluid culture with Matriks Assisted Laser Desorption/ Ionization - Time of Flight (MALDITOF) technology. Then oral clarithromycin was added to treatment for 10 days. Intraperitoneal cefepime was given for 14 days. At the end of therapy, peritoneal fluid culture was negative. During the one-year follow-up, he did not have any peritonitis attacks.

\section{DISCUSSION}

Peritonitis is the most common complication of peritoneal dialysis. In 2015, 19 children with peritoneal dialysis were followed in our hospital and peritonitis occured in 3 of them. Annual incidence of peritonitis in our hospital in 2015 was calculated 0.013 attack/patient month.

The causative microorganisms are frequently bacteria. Although 50 percent of cases are caused by gram positive microorganisms (S. aureus, coagulase-negative staphylococci and streptococci), gram-negative microorganisms (E. faecalis and E. coli) are detected in 15 percent of patients with peritonitis. In addition; peritoneal fluid culture is negative in 20 percent of infections. ${ }^{1}$

Campylobacter is a small, gram-negative microaerophilic bacteria. It presents in intestinal tracts of animal hosts, especially in poultry. Campylobacter infections may be transmitted to humans via animals, animal products and water. Campylobacter jejuni is the most responsible pathogenes (60\%) following Campylobacter fetus (20\%). ${ }^{4}$ Clinical symptoms may be diarrhea, fever, abdominal pain, and vomiting. Few cases with Campylobacter peritonitis have been reported in the literature. It is difficult to identify this pathogen clinically due to the absence of specific symptoms for this microorganism.

Some studies suggested that diarrhea occurs more frequently in Campylobacter peritonitis. ${ }^{5,6}$ Diarrhea may be a predisposing factor. Patients with Campylobacter gastroenteritis may carry the microorganism on their skin and hands and may cause infection by contamination of peritoneal dialysis. Another way is transmural migration from the inflamed intestine. ${ }^{6}$

Campylobacter spp. are resistant to beta lactam antibiotics and some cephalosporins. Peritonitis guidelines recommend that cephalosporins should be used for first choice treatment but they could be resistant to Campylobacter spp. On the other hand macrolides are more effective treatment modalities. ${ }^{7}$
Although some adult cases have been reported previously, this is the first pediatric case in the literature. Elshafie et al. reported 3 cases with Campylobacter peritonitis. One of them was 64-year-old woman who had got abdominal pain and non-bloody diarrhea. Campylobacter coli was isolated from peritoneal dialysate culture. Campylobacter spp. were not detected in stool cultures. Another case was 61-year-old diabetic man who was treated with CAPD. He had got abdominal pain, diarrhea, vomiting and fever. Although stool cultures were negative, Campylobacter fetus was isolated from peritoneal dialysate culture. Last case was 77-year-old woman with chronic glomerulonephritis. She admitted to hospital with abdominal pain, vomiting and watery diarrhea. Campylobacter lari was isolated from peritoneal dialysate culture. All three cases were treated with intraperitoneal cefazolin. ${ }^{4}$ Unlike these cases, our patient did not have any history of acute gastroenteritis.

In another report 38-year-old male with CAPD, was presented with acute peritonitis symptoms. Campylobacter jejuni was isolated from peritoneal fluid culture. The patient was successfully treated with oral clarithromycin for 12 days. ${ }^{7}$ Ma et al. reported the largest case series of Campylobacter peritonitis. Twelve cases were treated with intraperitoneal cefazolin and ceftazidime with or without addition of vancomycin or gentamisin. Six patients who were unsuccessfully treated with empiric antibiotics, completely recovered after using an oral macrolide. Thus, Campylobacter jejuni peritonitis seems to improved with oral clarithromycin therapy. ${ }^{6,7}$ Our patient was also successfully treated with oral clarithromycin, besides we did not see any side effects.

In conclusion, Campylobacter peritonitis is a rare infection in children. Oral clarithromycin seems to be more effective for treatment. If appropriate therapy is started immediately, clinical outcomes will be satisfactory. Although adult cases have been reported in the literature, Campylobacter spp. must be remembered as a causative microorganism for peritonitis in children.

\section{REFERENCES}

1. Port FK, Held PJ, Nolph KD, Turenne MN, Wolfe RA. Risk of peritonitis and technique failure by CAPD connection technique: a national study. Kidney Int 1992;42(4):967-74.

2. Warady BA, Bakkaloglu S, Newland J, Cantwell M, et al. Consensus guidelines for the prevention and treatment of catheter-related infections and peritonitis in pediatric patients receiving peritoneal dialysis: 2012 update. Perit Dial Int 2012;32(Suppl 2):S32-86. 
3. Li PK, Szeto CC, Piraino B, Bernardini J, et al. Peritoneal dialysis-related infections recommendations: 2010 update. Perit Dial Int 2010;30(4):393-423.

4. Elshafie SS, Asim M, Ashour A, Elhiday AH, et al. Campylobacter peritonitis complicating continuous ambulatory peritoneal dialysis: report of three cases and review of the literature. Perit Dial Int 2010;30(1):99-104.

5. Wood CJ, Fleming V, Turnidge J, Thomson N, Atkins RC. Campylobacter peritonitis in continuous ambulatory peritoneal dialysis: report of eight cases and a review of the literature. Am J Kidney Dis 1992;19(3):257-63.

6. Ma TK, Lee KP, Chow KM, Pang WF, et al. Campylobacter peritonitis complicating peritoneal dialysis: a review of 12 consecutive cases. Perit Dial Int 2013;33(2):189-94.

7. VerbanckJJ, Verlinde AM, Verbanck MI, Claessens MM, et al. Campylobacter jejuni II peritonitis in a CCPD patient: cure by oral clarithromycin. Perit Dial Int 1999;19(1):85-6. 